\title{
Controlling Springback With Compensation Strategies
}

\author{
Jochen Weiher, Bert Rietman, Kim Kose, Stephan Ohnimus, Martin Petzoldt
}

\author{
INPRO Innovationsgesellschaft für fortgeschrittene Produktionssysteme in der Fahrzeugindustrie mbH \\ Hallerstraße 1, 10587 Berlin, Germany
}

\begin{abstract}
Several measures are applied in order to reduce springback but it is not always possible to reduce springback sufficiently. Therefore techniques have been developed to counter springback by modification of the tool shape (overbending). In this paper the smooth displacement adjustment method (SDA) for springback compensation is presented. The discrete springback displacement field is approximated by an L2-projection of suitable continuous smooth functions. Multiplied by a compensation factor the approximating continuous displacement field is applied to the tool surface including the addendum. An application of the new method on an industrial part is demonstrated. The main advantage is the easy handling of the method.
\end{abstract}

\section{INTRODUCTION}

Geometrical inaccuracies of sheet metal parts due to springback after forming operations are the reason for considerable efforts in the tool and process development. Although some amount of springback occurs after every deep drawing its major cause can be identified with the increased application of high and ultra high strength steels and aluminum. These modern light-weight materials feature a high ratio of yield stress to Young's modulus. The increased part size adds up in making springback an urging problem. To cope with the problem of springback different techniques have been developed. The first technique is the variation of process parameters like blank holder force or the use of different draw beads. For structural parts stiffeners or beads can be added to change the stiffness of the part. An additional calibration operation can be used. The last and most complex technique is the modification of the tool geometry, in order to overbend the part and therefore compensate the springback. This method is expensive and time intensive as largely based on workshop knowledge [2].

In this paper reasons of springback are discussed followed by an overview of different springback control strategies including a new method, the smooth displacement adjustment method (SDA). The new method is an extension of the original displacement adjustment method (DA). Its main idea is the introduction of a continuous smooth displacement function compensating the complete tool geometry including the addendum and the blank holder. With appropriate $\mathrm{CAD}$ algorithms it opens the road towards modification of CAD data. This new method is demonstrated on an actual car part showing very good results.

\section{Springback In The Process Chain}

Springback occurs in each stage of the production process. The reason is that the material undergoes changes which affect its geometry, geometrical stiffness and residual stresses. This means springback is not only a phenomenon of the deep drawing operation but also of the subsequent trimming, flanging and hemming operations. Thus even parts which have a satisfactory geometry after the first forming operation can finally be geometrically inaccurate.

Generally springback has to be compensated in the causing forming operation. As an exception springback due to trimming has to be compensated in the previous forming operation. Springback differs between different parts and materials therefore it is not possible to give a general rule for its compensation. Specific strategies can only be given for parts which are geometrically similar. 


\section{FE-Simulation}

Compensating springback is an iterative process. Doing this with real tools is very cost-intensive. The numerical simulation of the deep drawing process is standard in industrial practice. This is not true for determining springback. The numerical simulation of springback is only of good quality if done very carefully and requires more time than the deep drawing simulation.

Usually the FE-simulation in sheet metal forming is performed using linear 3-node or bilinear 4-node elements with five or seven integration points in the thickness direction and reduced or full integration in the plane. The quality of the results is good with respect to the thickness and the punch forces and the draw-in but of minor quality with respect to the stresses or the part form after springback. This is due to the insufficient quality of the calculated stresses. Small element sizes help to get better results but lead to very long calculation times. The use of quadratic elements which could represent the geometry much better is avoided because there is no easy way to calculate the appropriate contact zones.

\section{COMPENSATION STRATEGIES}

Compensation of springback is understood as the modification of the original tool geometry, resulting in diminished shape deviations between target geometry and actual result of the forming operation.

Several approaches to control springback have been described in literature. Most known are the displacement adjustment (DA) [3,4] and the spring forward method (SF) [1]. All control strategies have in common that the aim is not to reduce springback but to obtain the target geometry after forming and springback. This is in contrast to methods like increasing the blank holder force which can reduce springback directly.

Compensation of springback is achieved by modifications of tool geometry. The basis is the part after forming either in reality or after numerical simulation of the springback. The result is then compared with the target geometry. In the DA-method the deviation of the resulting geometry after springback and the target geometry is measured first. A multiplication of this field with the compensation factor is then used as a shape modification. Depending on the geometry and the material this factor varies between -1 and -2.5 . A sequential application of the
DA-method enhances the compensation result significantly. The standard DA-method works on the tessellated surface and gives a point wise modification of the tool. In the SDA-method the tool gets changed by a continuous function which is not restricted to the part geometry. The SDA-method can be applied to change the CAD-data of the part and tools.

The SF-method starts with the forces acting on the punch in the end of the forming simulation. These forces are applied on the target geometry in a subsequent elastic FE-calculation resulting in a geometry which compensates the springback. In the SF-method it is assumed that residual stresses do not influence the springback behaviour.

This method is confined to compensate springback in the first forming operation whilst the DA-methods can also be applied to springback as the result of all forming steps.

\section{The Smooth Displacement Adjustment}

\section{The Displacement Adjustment Method}

The idea of the DA-method is intuitively straightforward and has been used by experienced engineers for some time. The definition of one iteration of the DA-method is as follows. The target position of a material point in the part is $\mathbf{t}$ and $\mathbf{S}$ its actual position after springback. The position of the compensated material point $\mathbf{c}$ is then defined by the following equation:

$$
\mathbf{c}=\mathbf{t}+a(\mathbf{s}-\mathbf{t})
$$

where $a$ is an appropriate compensation factor. This compensation factor can be determined by trial and error and usually is in the range from -1 to -2.5 . This scheme can be iterated by repeating the compensation step [3].

The reason for the success of the DA-method stems from the fact that the deformation caused by springback is mainly elastic and linear with respect to modifications of the tool geometry. Therefore the deformations caused by springback can be canceled out by additional deformations due to the tool geometry. Note that in most parts a compensation factor $a=-1.3$ is used. This means that springback is not reduced by the compensation but increased. 


\section{Definition Of The Smooth Displacement Adjustment Method}

In the original DA-method the displacement caused by springback is applied directly to the part geometry. Usually the geometry after springback is either determined by numerical simulation or by photooptical methods. In both cases the springback field is defined only at discrete points of the part. These are the nodes of the discretized part or discrete points determined by the optical measurement. In the latter case there is no straightforward one to one relation between points of measurement and points on the target geometry. In these cases the springback displacement field cannot simply be applied to nodes of the tool geometry. To remedy this situation the SDA-method, which is a simple and robust algorithm for determining a continuous and smooth displacement field $\mathbf{u}(\mathbf{x})$, has been developed. The displacement field is therefore defined at all points of the tool geometry. It is not important if the tool geometry is described by a tessellated surface of triangles or quadrangles or by CAD-patches which are spanned between control points. A single iteration of the SDAmethod is defined as follows. Given a material point $\mathbf{x}$ the compensated material point $\mathbf{c}(\mathbf{x})$ is defined by

$$
\mathbf{c}(\mathbf{x}):=\mathbf{x}+a \mathbf{u}(\mathbf{x})
$$

where $a$ is again the compensation factor. Here $\mathbf{x}$ is a point on the geometry to be deformed and $\mathbf{u}(\mathbf{x})$ is the smooth displacement field defined for all point in the three dimensional space. Thus the continuous displacement field $\mathbf{u}(\mathbf{x})$ approximates and replaces the discrete field $(\mathbf{s}-\mathbf{t})$ of the DA-method. The task is now to find $\mathbf{u}(\mathbf{x})$ for which

$$
\mathbf{u} \sim \mathbf{s}-\mathbf{t}
$$

holds in all discrete points $\mathbf{t}$, meaning that the smooth displacement field differs only little from the discrete displacement field. The task is to find the displacement field $\mathbf{u}$ that minimizes

$$
\|(\mathbf{s}-\mathbf{t})-\mathbf{u}\|_{\mathbf{L}_{2}}
$$

Each space component of the discrete displacement field is approximated independently. Note that the deformation caused by springback is mostly an elastic deformation and therefore a relatively smooth deformation with long modes. In fact its description is simpler than the description of the part geometry itself. This justifies the approximation of springback with polynomials $p_{i}, i=1, \ldots, n$ of low order. The displacement field is described by the following ansatz

$$
\mathbf{u}(x, y, z)=\sum_{i} \mathbf{a}_{i} p_{i}(x, y, z)
$$

where the vector $\mathbf{a}_{i}$ contains the weights of the polynomials. Note that $\mathbf{u}$ and $\mathbf{a}_{i}$ are vectors in the three dimensional space.

Inserting (5) into equation (4) gives after a variation the linear system of equations

$$
\sum_{i} m_{i j} \mathbf{a}_{i}=\mathbf{r}_{j}
$$

with the matrix entries

$$
m_{i j}=\int_{G} p_{i} p_{j} d x
$$

where the domain of integration $\mathrm{G}$ is the target geometry. The numerical integration is carried out by summarizing over all facets of the target geometry with appropriate mass lumping.

The right hand side $\mathbf{r}$ is given by the components of the discrete displacement field $(\mathbf{s}-\mathbf{t})$. Each component $\mathbf{r}_{j}$ is defined with

$$
\mathbf{r}_{j}=\int_{G}(\mathbf{s}-\mathbf{t}) p_{j} d x
$$

The approximation error (4) can be calculated and has to be small with respect to $\|\mathbf{u}\|_{\mathbf{L}_{2}}$. Another possibility is to calculate the maximum deviation between the discrete and the continuous displacement field. Our experiences with displacement fields from real industrial parts showed that the error is in the range of $10 \%$ and can be considered as small. It has to be pointed out that the level of error made by calculating springback and measuring shape deviation is of equal or higher order. If necessarily the function space can be extended further.

\section{Extensions And Restrictions}

Obviously the presented approach is not restricted to polynomials. Instead Bezier splines may also be suitable to represent the displacement field. Furthermore the approximating functions can be 
confined to have local support in order to restrict the compensation to local areas where springback is dominant. Another extension is to define a non constant compensation factor, for instance as a function of the length of $\mathbf{u}(\mathbf{x})$. Symmetry conditions can be regarded as well by restricting the space of polynomials.

The smooth displacement function is well defined only in a vicinity of the part. As polynomial functions tend asymptotically to infinity the smooth displacement function too takes large values outside the part. In some cases this can lead to a distortion of parts of the blankholder and affect the developability of the blankholder. These problems too can be avoided restricting by multiplying the polynomials with a cutoff function vanishing on the blankholder.

A serious restriction may be the demand to have no undercut parts of the tool, i.e. parts where the normal vector is not oriented towards the drawing direction. This restriction is due to the method of compensation itself and not a special property of the proposed algorithm.

Another important application of the SDA-method is its use when the discrete displacement field is not known. For instance when the optically measured part geometry after springback is given and has to be compared to the target geometry. In this case a discrete distance field between the two tessellated objects has to be calculated. This field can be very rough at regions with high curvature and can therefore seriously differ from the actual displacement field. The SDAmethod smoothes the rough field and can help to recover the original smooth displacement field.

\section{EXAMPLE}

The application of the SDA-method is demonstrated on a wall for a spare wheel well, see fig. 1 , with dimensions $1000 \mathrm{~mm}$ by $30 \mathrm{~mm}$ made of higher strength steel ZStE 340. The part is produced with a single deep drawing operation followed by one flanging operation. Especially the flanging operation showed large shape deviations, because of non developable flanges being drawn. The shape deviation was up to $7 \mathrm{~mm}$ and the compensation required a compensation factor of -2.5 .

The whole production process was simulated with the implicit FE-code INDEED. The numerically computed springback was reported to be in good accordance with the practically observed. Springback occurred mainly at the two ends of the flange.

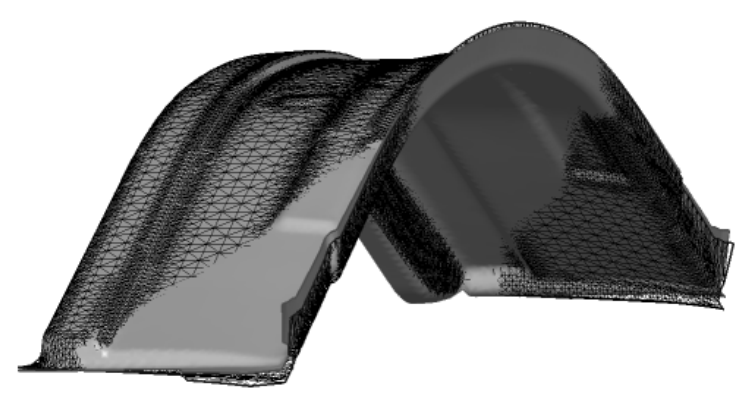

FIGURE 1. Shape deviation up to $7 \mathrm{~mm}$ with original tool geometry.

The compensation algorithm was tested first. The approximation with the calculated continuous displacement field $\mathbf{u}(\mathbf{x})$ was checked against the discrete displacement field of the springback. To do this the continuous displacement field $\mathbf{u}(\mathbf{x})$ was applied to the part geometry before springback and compared to the geometry after springback. Figure 2 shows that both geometries are in good accordance.

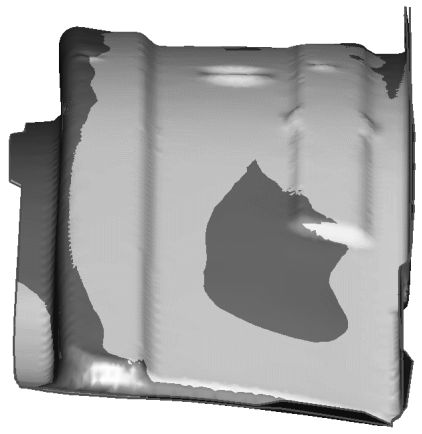

FIGURE 2. Part geometry after springback (light grey) and after application of smooth displacement field (dark grey) in good accordance (both displacement fields are enlarged by the factor 5)

Giving good results the displacement field was applied to all parts of the tool geometry (punch, blankholder, die), see fig. 3. This resulted in a smooth deformation of the original tool geometry. Note that the whole tool was compensated smoothly, even in regions of the addendum. 


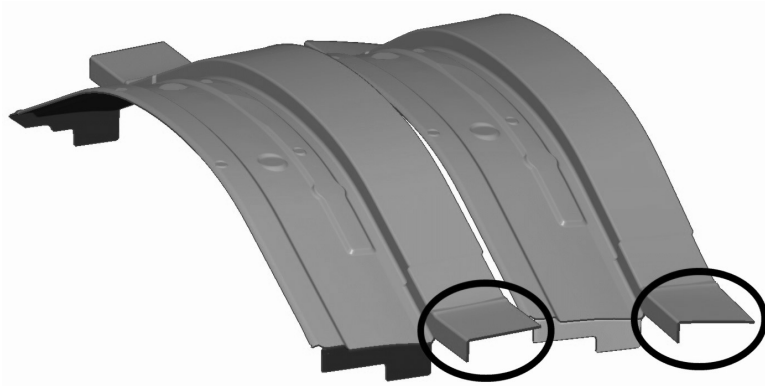

FIGURE 3. Tool geometries: left original, right compensated, note the smoothly compensated flanges.

Finally the whole forming simulation was carried out with this compensated tool geometry. The shape deviation after springback was significantly smaller and not larger than $1.5 \mathrm{~mm}$ down from previously $7 \mathrm{~mm}$, see fig. 4 .

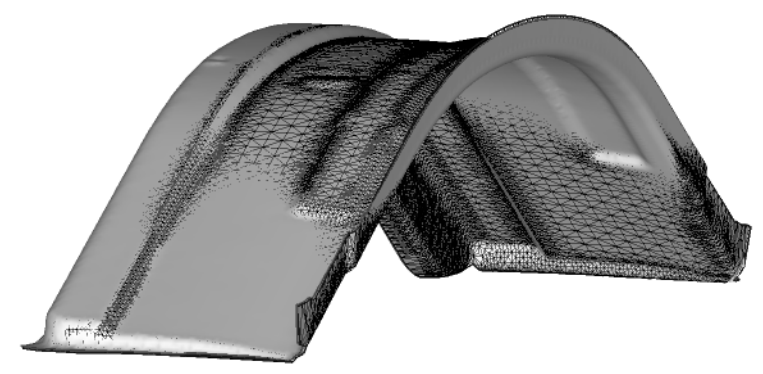

FIGURE 4. Shape deviation $1.5 \mathrm{~mm}$ with compensated tool geometry.

\section{CONCLUSIONS AND FUTURE RESEARCH}

The SDA-method presented in this paper is an extension of the standard DA-method. It has been successfully demonstrated on an industrial part. It is characterized by the potential of further enhancement and refinement.

As mentioned before springback controlling methods are independent of the springback simulation algorithms. Nonetheless a good and exact springback simulation will help considerably in avoiding shape deviations because a simulation is significantly less cost-intensive than the manufacturing and altering of real tools. Elements which can give a better geometry and stress approximation using quadratic functions may help a lot in achieving this.

\section{ACKNOWLEDGMENTS}

The authors wish to express their thanks to KUKA Werkzeugbau Schwarzenberg GmbH and DaimlerChrysler AG Germany for providing comparisons between simulations and measurements on real parts.

\section{REFERENCES}

1. Karafillis, A.P. and Boyce, M.C., "Tooling design in sheet metal forming using springback", Int. J. Mach. Tools. Manuf. 34, 113 (1992)

2. Roll, K. and Bogon, P., "Rückfederung als Genauigkeitshindernis - notwendige Weiterentwicklungen der Simulationstechnik" in Proceedings Sächsische Fachtagung Umformtechnik 2003, pp. 89-108 (in german)

3. Wagoner, R., „Fundamental aspects of springback in sheet metal forming" in Proceedings Numisheet 2002 edited by D. Yang et.al., pp 13-19, 2002

4. Wagoner, R., "Design of sheet forming dies for springback compensation" in Proceedings ESAFORM 2003, edited by V. Brucato, 2003, pp. 7 - 14 